\title{
Predictors of Progression-Free Survival and Overall Survival in Metastatic Non-Clear Cell Renal Cell Carcinoma: A Single-Center Experience
}

\author{
Sasanka Kumar Barua ${ }^{a}$, Yashasvi Singh ${ }^{a}$,, Saumar Jyoti Baruah ${ }^{a}$, Rajeev T.P. ${ }^{a}$, \\ Puskal K. Bagchi ${ }^{\text {a }}$, Debanga Sarma ${ }^{\text {a }}$, Mandeep Phukan ${ }^{\mathrm{a}}$
}

\begin{abstract}
Background: Due to the infrequency of non-clear cell renal cell carcinoma (RCC), there is currently a paucity of high-quality literature to help guide the effective treatment of these tumors. Recently, biomarkers such as platelet to lymphocyte ratio (PLR), lymphocyte to monocyte ratio (LMR), systemic immune inflammation (SII) index and C-reactive protein to albumin ratio (CAR) have been demonstrated to be closely related to poor prognosis of patients with RCC. The objective of this study was to evaluate these biomarkers for determining the progression-free survival (PFS) and overall survival (OS) in patients with metastatic non-clear cell cancer.
\end{abstract}

Methods: We retrospectively reviewed 31 cases diagnosed with metastatic non-clear cell RCC from January 2012 to December 2017. We assessed the prognostic value (OS and PFS) of pretreatment PLR, LMR, SII index and CAR based on multivariate analysis and KaplanMeier survival curve.

Results: Median time of OS and PFS were 15.5 months (95\% confidence interval (CI): 13.7 - 15.2) and 10.9 months (95\% CI: 8.9 - 12.8), respectively. The median PFS $(0.001)$ and OS $(\mathrm{P}=0.01)$ was shorter in patients with PLR $>171$, LMR $<2.61$. Moreover, median PFS but not OS was significantly lower in SII index $>883(\mathrm{P}=0.064)$ and CAR $>0.11(\mathrm{P}=0.229)$. Scan to surgery time (3.91 weeks, $\mathrm{P}=0.001)$ was also significantly related to progression.

Conclusions: Elevated pretreatment inflammatory biomarkers such as PLR, LMR, SII index and CAR are significant determinants of shorter PFS and OS (PLR and LMR only) in patients with metastatic non-clear cell RCC treated with cytoreductive nephrectomy.

Keywords: Non-clear cell RCC; Progression-free survival; Overall

Manuscript submitted February 18, 2019, accepted March 29, 2019

${ }^{a}$ Department of Urology, Gauhati Medical College Hospital, Guwahati, Assam, India

${ }^{\mathrm{b} C o r r e s p o n d i n g ~ A u t h o r: ~ Y a s h a s v i ~ S i n g h, ~ D e p a r t m e n t ~ o f ~ U r o l o g y, ~ G a u h a t i ~}$ Medical College Hospital, 3rd Floor, GMCH Complex, GMC Hostel Road, Bhangagarh, Guwahati, 781032 Assam, India.

Email: yashasvisingh075@gmail.com

doi: https://doi.org/10.14740/wjon1188 survival; PLR, LMR; SII index; CAR

\section{Introduction}

Although many breakthroughs have been achieved in the management of metastatic renal cell carcinoma (RCC), the majority of studies to date have been limited to patients with a clear cell variant. RCC, the seventh most common malignancy for men and the ninth for women the world over, represents $2-3 \%$ of all malignances in adults [1]. Due to the infrequency of non-clear cell RCC, there is currently a paucity of highquality literature to help guide the effective treatment of these tumors. Cytoreductive nephrectomy (CNT) represents the recommended standard of care in metastatic RCC and it is one of the available validated tools to combat metastatic non-clear cell RCC at present [2]. Notwithstanding the advancement in surgical techniques, immunotherapy and targeted treatment in managing these cases, the long-term survival still remains unsatisfactory largely because of high in bed recurrence, distant metastasis and poor response to chemoradiotherapy [3, 4]. Although postoperative histopathology information is presently the most accepted factor for patient stratification, these parameters may not be entirely dependable for guiding adjuvant treatment in metastatic non-clear cell RCC. Additionally, since most prognosis predictors are assessed postoperatively, preoperative biomarkers are needed to early predict oncologic outcomes. The identification of preoperative biomarkers may have clinical utility to guide therapeutic decisions and followup treatment. More and more evidence supports that inflammation exerts a crucial role in the pathogenesis and progression of various malignances, including non-clear cell RCC [5-7].

Circulating biomarkers, signifying inflammation, are believed to be potential prognostic factors for RCC patients. Proinflammatory cytokines and immune-inflammatory circulating cells (neutrophils, lymphocytes and platelets) seem to play a role in promoting cancer cell proliferation and invasion [8]. Platelet to lymphocyte ratio (PLR) and lymphocyte to monocyte ratio (LMR), two inflammatory markers which come from the blood cells, have gained prognostic value in a number of malignant diseases, including RCC [9]. A number of researches have examined their role as prognosis predictors, 
nevertheless, the coherence and importance of the prognostic value of PLR and LMR are still needed to be explored. A new inflammatory index, the systemic immune inflammation (SII) index, based on neutrophil, lymphocyte and platelet counts, has been recently found to be associated with poor outcome in patients with hepatocellular carcinoma (HCC) [10]. The rationale of this new index is based on the combination of three factors independently related to prognosis in some cancers and was thought to have a stronger prognostic power than any of the factors analyzed alone. In recent times, preoperative Creactive protein/albumin ratio (CAR) has been reported as an independent prognostic marker in $\mathrm{HCC}$, gastric cancer (GC) and small-cell lung cancer (SCLC) [11]. Although a recent study reported the prognostic influence of CAR on overall survival (OS) of patients with clear cell renal carcinoma [12], its prognostic role in metastatic non-clear cell RCC still needs to be further explored.

In this present retrospective study, we aim to achieve a better contemporary patient selection protocol based on preoperative circulating biomarkers such as PLR, LMR, SII index and CAR in patients of metastatic non-clear cell RCC to achieve greater progression-free survival (PFS) and OS benefit after CNT and to develop a prognostic model based on these inflammatory biomarkers for the same.

\section{Patients and Methods}

We performed a retrospective cohort study of 31 consecutive metastatic non-clear cell RCC patients who underwent CNT between January 2012 and December 2017 at the Department of Urology and Renal Transplantation after obtaining institutional ethical committee clearance. The inclusion criteria were as follows: 1) Patients were histologically diagnosed with nonclear cell RCC with radiological investigation showing at least one site of metastasis to either lung bone or brain; 2) Data on complete blood laboratory measurements relaying information on PLR, LMR, SII index and CAR done within 1 week before performing CNT. Patients without adequate blood reports prior to surgical resection and patients with active inflammatory disease were excluded from the study. The baseline clinical, pathologic and biochemical parameters were collected, including age at the time of surgery, gender, tumor size, tumor stage ( $\mathrm{T}$ stage), tumor necrosis (TN), microscopic invasion (MI, HPE), number of positive lymph nodes (LNs) on HPE, scan to surgery time (SST), neutrophil count, lymphocyte count, monocyte count, platelet count, CRP and albumin level by using a standard data extraction system. T stage was determined based on the 2010 TNM classification of malignant tumors staging system and tumor grade was defined according to the Fuhrman grading system. The PLR, LMR, SII index (platelet count $\times$ neutrophil count/lymphocyte count) and CAR were calculated.

A 3 monthly ultrasonography (USG) for whole abdomen with Doppler study, high-resolution computed tomography (HRCT) for thorax and when required multidetector computed tomography (MDCT) and intravenous urography (IVU) in cases of positive USG findings were performed tailored to patient performance and clinical features in the first 2 years. Due to high aggressiveness of disease and heavy metastatic burden, a low-dose contrast-enhanced computed tomography (CECT) protocol was requested to assess the in bed recurrence or increase in metastatic site. Data were summarized by frequency for categorical variables and by median and range for continuous variables. Association between categorical variables was assessed using the Chi-square or Fisher's exact test, when appropriate. Differences were considered statistically significant when $\mathrm{P}<0.05$. PFS was calculated from the start of first-line treatment until disease progression or last follow-up. OS was calculated from the start of first-line treatment until death or last follow-up. The Kaplan-Meier survival curve was used to estimate PFS and OS. The log rank test and cox proportional hazard regression were used to test for differences between groups. A multivariate analysis was carried out by cox regression model. Estimated hazard ratios (HRs), their 95\% confidence intervals (95\% CIs) and P values were calculated from the Cox proportional hazard regression model. The optimal cut-off points for the inflammation-based factors were determined by receive operating characteristic (ROC) analysis and the areas under the curve (AUCs) were calculated. Statistical analyses were performed using IBM SPSS 21.0 software.

\section{Results}

There were a total of 31 cases of metastatic non-clear cell RCC included in the retrospective analysis with the special emphasis on PFS and OS. All cases belonged to multi-ethnic society residing in the eight North Eastern states of India. The male to female ratio in the study was 2.1 to 1 with 21 males and 10 females with mean age for men being $58.12 \pm 4.25$ years and mean age for women being $53.17 \pm 3.5$ years. The male population was mostly dispensable towards progression as compared with the female population though not significant $(\mathrm{P}$ $=0.373$, Table 1). The disease load was more on the left side $(\mathrm{n}=18,58.1 \%)$ as compared to the right side $(\mathrm{n}=13,41.9 \%)$ with almost similar progression rate $(61.1 \%$ vs. $61.5 \%, \mathrm{P}=$ $0.981)$. The median follow-up time was $66 \pm 5.8$ weeks with an inability to follow-up for a consecutive of 6 weeks leading to expulsion from analysis. Eleven patients (35.48\%) were lost in the study due to extensive disease profile and related cardiac events. Nineteen patients $(61.29 \%)$ had an event related to progression (increase in metastatic lesions in 13 cases and resection site recurrence in six cases) diagnosed on a followup USG W/A and confirmed on a CECT W/A + Pelvis. From a histology point of view, papillary and unclassified variant, each contributed $29 \%$ in the study along with chromophobe and collecting duct RCC constituting $19.4 \%$ and $22.6 \%$, respectively. Maximum number of progressions was recorded in the papillary and the unclassified variants with $100 \%$ strike rate and none recorded in the collecting duct histology. This observation was significantly associated $(\mathrm{P}=0.001)$ with the PFS but not significantly correlated with the same $(\mathrm{P}=0.810)$ (Table 1). The metastatic load consisted of oligometastatic (< 3 sites) non-clear cell RCC in 20 cases and polymetastatic $(\geq 3$ sites) non-clear cell RCC in 11 cases. The most common site of metastasis was lung followed by liver and bone. The distribu- 
Table 1. Epidemiological and Clinicopathological Data in the Progression and Non-Progression Group

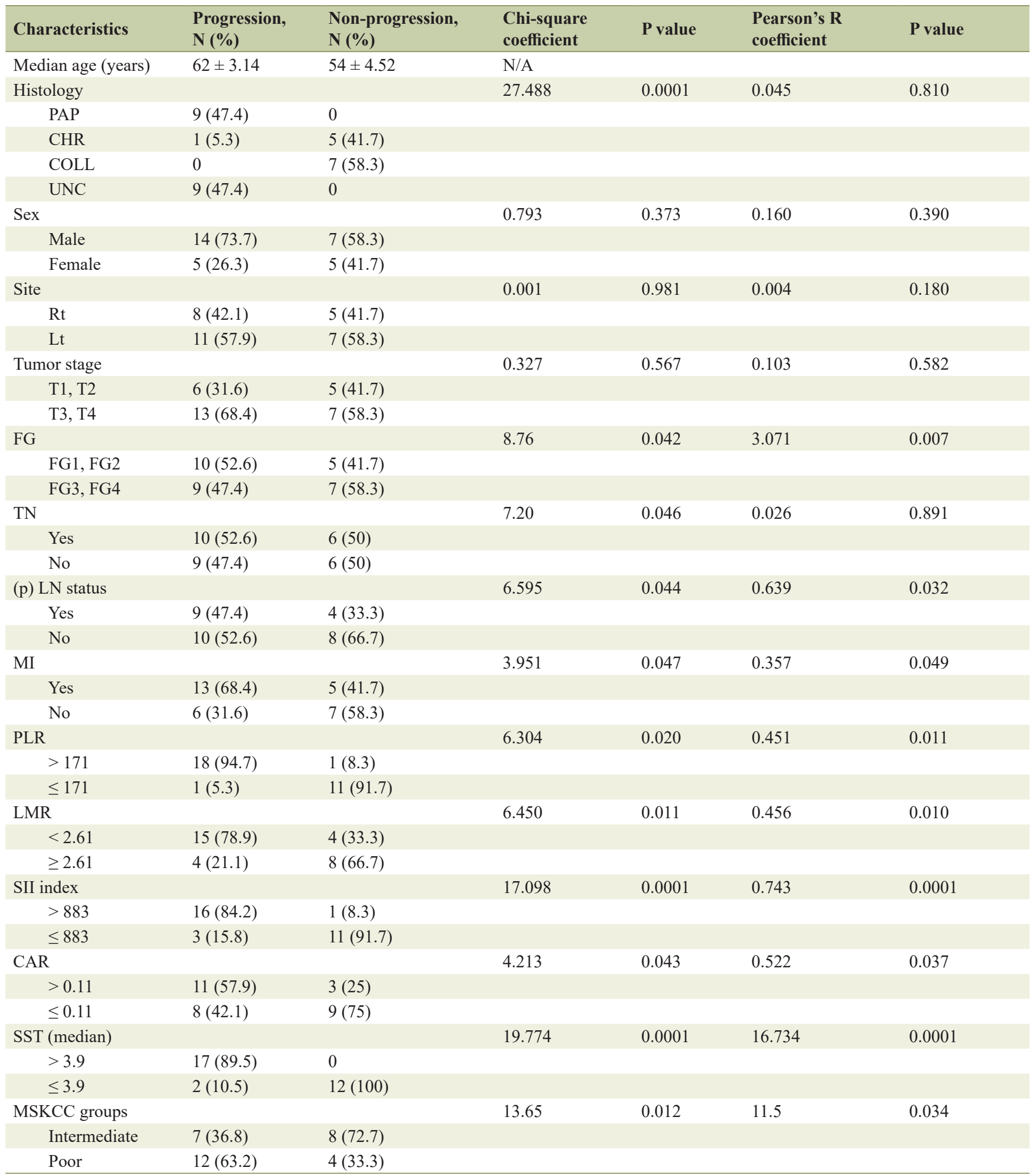

The percentage value depicted denotes the value within the respective progression or non-progression groups. FG: Fuhrman nuclear grade; TN: tumor necrosis; LN: lymph node; MI: microscopic invasion; PLR: platelet to lymphocyte ratio; LMR: lymphocyte to monocyte ratio; SII: systemic immune inflammation; CAR: C-reactive protein/albumin ratio; SST: scan to surgery time; MSKCC: Memorial Sloan Kettering Cancer Center. 

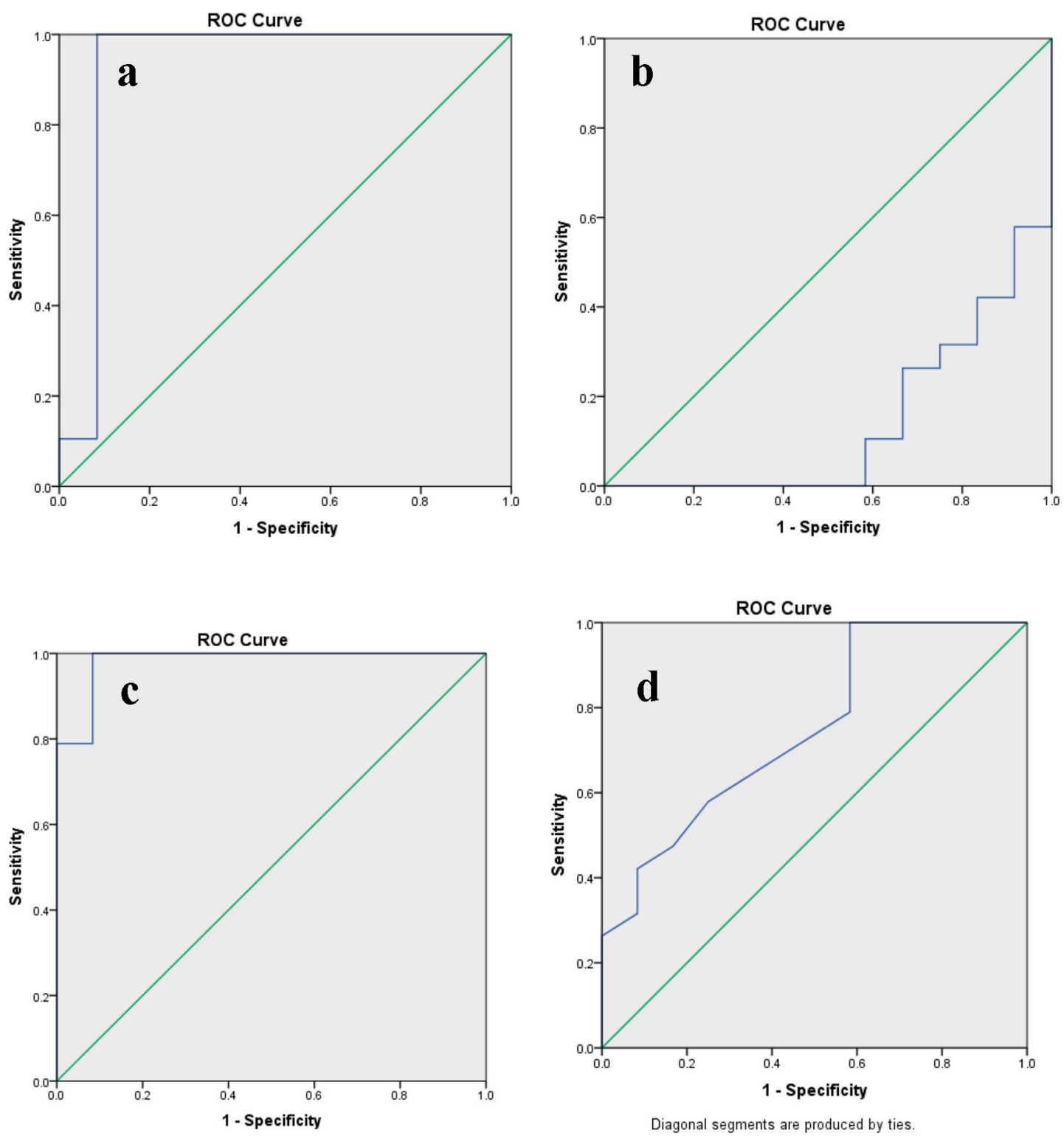

Figure 1. The predictive ability of ROC curve to determine the cut-off point for (a) PLR, (b) LMR, (c) SII index and (d) CAR in relation to disease progression. ROC: receive operating characteristic; PLR: platelet to lymphocyte ratio; LMR: lymphocyte to monocyte ratio; SII: systemic immune inflammation; CAR: C-reactive protein/albumin ratio.

tion of bone metastasis was mainly concentrated on dorsolumbar spine than appendicular skeleton. After CNT, there was an emergence of additional metastasis in 13 cases which were mainly in liver $(n=7)$ and dorsolumbar spine $(n=6)$. A higher T stage (T3, T4) was observed in 20 cases $(64.5 \%)$ with $68.4 \%$ progression rate $(\mathrm{n}=13, \mathrm{P}=0.567)$ for higher $\mathrm{T}$ stage (Table 1). The odds ratio (OR) for an event (progression) occurring for higher T stage was 0.839 (95\% CI: 0.448 - 1.573). Similarly when evaluating the Fuhrman nuclear grade (FG), it was perceived that a higher grade (FG3, FG4) was significantly associated and correlated with number of progressions (Table 1). The OR for the same was 1.185 (95\% CI: 0.676 - 2.077).

Radiological characterization of all renal masses was also done with optimal metabolic evaluation. TN was present in $51.6 \%$ cases and was significantly associated with the progression rate $(\mathrm{P}=0.046)$. Ten cases $(62.5 \%)$ progressed at an interval of 12 weeks within the TN group $(\mathrm{n}=16$, Table 1$)$ with OR being 0.900 (95\% CI: $0.212-3.822)$. Tumor size with greatest dimension $\geq 7 \mathrm{~cm}$ (median size: $8.25 \pm 2.12 \mathrm{~cm}$ ) was also assessed in the study with 15 cases having crossed the cut-off limit and was significantly associated $(\mathrm{P}=0.001)$ and correlated $(0.03)$ with the progression rate. Total number of positive LNs were also looked upon for each case and in out of the total 31 cases, $41.9 \%$ cases $(n=13)$ had positive LNs in final biopsy report. A total of nine out of 13 cases $(69.2 \%)$ with positive LNs progressed in the final analysis $(\mathrm{P}=0.044)$ 
Table 2. Multivariate Analysis for Variable Considered for PFS and OS

\begin{tabular}{|c|c|c|c|c|c|c|}
\hline \multirow{2}{*}{ Characteristics } & \multicolumn{3}{|c|}{ PFS } & \multicolumn{3}{|c|}{ OS } \\
\hline & $95 \%$ CI & HR & $P$ value & $95 \%$ CI & HR & $P$ value \\
\hline Age (years) & $0.569-1.073$ & 0.781 & 0.127 & $0.247-1.316$ & 0.570 & 0.188 \\
\hline FG & $0.847-28.416$ & 15.51 & 0.065 & $0.157-22.150$ & 15.922 & 0.151 \\
\hline $\mathrm{TN}$ & $9.671-25.349$ & 15.65 & 0.005 & $0.033-5.789$ & 4.366 & 0.303 \\
\hline PLR & $1.102-1.460$ & 1.269 & 0.001 & $0.865-1.933$ & 1.293 & 0.045 \\
\hline LMR & $0.253-8.368$ & 1.456 & 0.001 & $0.713-16.101$ & 8.667 & 0.001 \\
\hline SII index & $1.005-1.040$ & 1.023 & 0.010 & $0.992-1.043$ & 1.017 & 0.183 \\
\hline CAR & $0.564-6.578$ & 4.445 & 0.001 & $0.076-1.763$ & 1.002 & 0.554 \\
\hline
\end{tabular}

PFS: progression-free survival; OS: overall survival; FG: Fuhrman nuclear grade; TN: tumor necrosis; LN: Iymph node; MI: microscopic invasion; PLR: platelet to lymphocyte ratio; LMR: Iymphocyte to monocyte ratio; SII: systemic immune inflammation; CAR: C-reactive protein/albumin ratio; SST: scan to surgery time; $\mathrm{Cl}$ : confidence interval; HR: hazard ratio.

with OR being 0.802 (95\% CI: 0.463 - 1.390). MI was present in a total of $58.1 \%(\mathrm{n}=18)$ cases with significant association $(\mathrm{P}=0.047)$ and correlation $(0.049)$ with the progression rate and OR standing at 0.308 (95\% CI: 0.079 - 1.194) (Table 1).

All blood parameters were done at 1 week prior to CNT and the results of PLR, LMR, SII index and CAR were matched with progression events both as a continuous and categorical variable. Based on the AUC of $0.925(\mathrm{P}=0.001)$ for PLR in the ROC analysis, the optimal cut-off was 171 (Fig. 1a). An elevated PLR ratio ( $>171$ ) was seen in $61.3 \%$ cases $(n=19)$ with $94.7 \%(n=18)$ cases experiencing a significant event (progression) in the same $(\mathrm{P}=0.01)$. Similarly, the cut-off for LMR was 2.61 based on the AUC of 0.140 (P $=0.001$ ) for the same in ROC analysis (Fig. 1b). A baseline LMR of $<2.61$ was seen in 19 cases $(61.3 \%)$ with 15 cases $(78.9 \%)$ suffering from an event in the progression group $(\mathrm{P}=$ $0.011)$. The cut-off value of SII index stood at 883 established upon AUC of $0.982(\mathrm{P}=0.001)$ for PFS in the ROC analysis (Fig. 1c) with the value of CAR being 0.11 , constructed upon an AUC of $0.750(\mathrm{P}=0.021)$ for the same (Fig. 1d). An elevated CAR $(>0.11)$ was seen in $45.2 \%$ cases $(n=14)$ with $78.6 \%(n=11)$ cases experiencing a significant event (progression) in the same $(\mathrm{P}=0.001)$. A standout value of SII index $(>883.29)$ was seen in 17 cases $(54.8 \%)$ with significant association $(\mathrm{P}=0.01)$ and correlation $(0.001)$ with the progression events $(94.1 \%, \mathrm{n}=16)$ (Table 1$)$. The median SST of $>3.9$ weeks was significantly correlated and associated with progression rate $(n=17,89.5 \%$, Table 1$)$. When the study population was distributed in terms of Memorial Sloan Kettering Cancer Center (MSKCC) risk protocol, none of the cases ferried in favorable risk group, while 15 cases landed in intermediate risk group and 16 cases were allotted to poor risk category. A total of seven cases progressed in the intermediate risk profile, while 12 cases progressed in the poor risk category (Table 1 ).

Table 2 shows the results of the multivariate analysis of
PFS and OS with the biochemical and clinicopathological parameters assessed in the study. It is clear from the previous analysis that the PLR, LMR, SII index and CAR are associated with the PFS of metastatic non-clear cell RCC patients (Table 1). After excluding the non-significant variables, the significant variables (p) LN status, FG, TN, MI, PLR, LMR, SII index and CAR along with SST were tested in the multivariate Cox proportional hazard model to find the determinants of PFS and OS. The multivariate analysis indicated that the TN (HR: 15.65; 95\% CI: $9.671-25.349 ; \mathrm{P}=0.005)$ along with $(\mathrm{p}) \mathrm{LN}$ status (HR: 14.67; 95\% CI: $4.811-24.781 ; \mathrm{P}=0.012$ ) and SST (HR: $0.41 ; 95 \%$ CI: $0.006-0.311 ; \mathrm{P}=0.002$ ) were an independent prognostic factor for PFS but not OS (Table 2).

\section{Impact of PLR in OS and PFS}

The multivariate analysis indicated that a high PLR $(>171)$ was related to a decreased PFS (HR: 1.269, 95\% CI: 1.102 $1.460, \mathrm{P}=0.001)$ and $\mathrm{OS}(\mathrm{HR}: 1.293,95 \% \mathrm{CI}: 0.865-1.933$, $\mathrm{P}=0.045$ ) (Table 2). In the poor risk MSKCC cohort with PLR $>171$, the estimated mean PFS stood at $6.4 \pm 2.4$ months $(\mathrm{P}=$ $0.012)$, while the estimated mean OS was $11.5 \pm 3.8$ months $(\mathrm{P}$ $=0.06)$. Similarly, intermediate risk category with PLR $>171$ had an estimated mean PFS of $8.3 \pm 2.6$ months $(\mathrm{P}=0.042)$, while the estimated mean OS stood at $13.7 \pm 4.3$ months $(\mathrm{P}$ $=0.036)$. In a recent study, it was seen that patients with a high PLR experienced a shorter RFS (HR: 2.64, 95\% CI: 1.35 $5.14, \mathrm{P}=0.004$ ) [13]. Our findings also showed that cases having increased PLR were prone to experience a higher $\mathrm{FG}(\mathrm{P}=$ $0.035), \mathrm{TN}(\mathrm{P}=0.041)$, a larger primary tumor size $(0.03)$ and a higher pathological T stage $(\mathrm{P}=0.005)$. The estimated mean OS for cases with PLR $\leq 171$ was significant at 18.8 months with standard error (SE) being 2.47 and $95 \%$ CI of $17.6-20.1$ $(\mathrm{P}=0.001)$ and the mean PFS for the same was significant at 16.2 months (95\% CI: 14.6 - 17.7, SE: 2.93, $\mathrm{P}=0.001)$. 


\section{Impact of LMR in OS and PFS}

Similarly, we found that a low LMR $(<2.61)$ was associated with inferior OS (HR: 8.667, 95\% CI: 0.713 - 16.101, P $=0.001)$ and it obtained significance on multivariate analysis. Furthermore, the association between a decreased LMR and PFS (HR: 1.456, 95\% CI: 0.253 - 8.368, P = 0.001) was also significant and resulted in a decreased PFS (Table 2). In the poor risk MSKCC cohort with $\mathrm{LMR}<2.61$, the estimated mean PFS stood at $8.4 \pm 3.1$ months $(P=0.01)$, while the estimated mean OS was $12.7 \pm 3.1$ months $(\mathrm{P}=0.03)$. Similarly, intermediate risk category with $\mathrm{LMR}<2.61$ had an estimated mean PFS of $9.5 \pm 1.8$ months $(P=0.005)$, while the estimated mean OS stood at $11.7 \pm 3.3$ months $(\mathrm{P}=0.021)$. Our findings also depicted that cases having decreased LMR were prone to experience a higher MI $(\mathrm{P}=0.03),(\mathrm{p})$ LN status $(\mathrm{P}=0.021)$ and a higher pathological $\mathrm{T}$ stage $(\mathrm{P}=0.01)$. The estimated mean OS for cases with $\mathrm{LMR} \geq 2.61$ was 18.3 months with SE being 2.98 and $95 \% \mathrm{CI}$ of $16.9-19.8(\mathrm{P}=0.035)$ and the mean PFS was 14.1 months (95\% CI: 11.8 - 16.4, SE: 4.61, P $=0.016$ ) for the same.

\section{Impact of SII index and CAR in OS and PFS}

In multivariate analysis, SII index more than the baseline ( $>$ 883) remained significant predictors of PFS (HR: $1.023,95 \%$ CI: $1.005-1.040, \mathrm{P}<0.010)$ but not OS (HR: 1.017, 95\% CI: $0.992-1.043, \mathrm{P}=0.183$ ) (Table 2). The estimated mean OS for cases with SII index $\leq 883$ was 17 months with SE being 5.25 and $95 \% \mathrm{CI}$ of $14.4-19.5(\mathrm{P}=0.064)$ but was not found to be significant and the mean PFS for the same was significant at 14.2 months $(95 \% \mathrm{CI}: 11.5-17, \mathrm{SE}: 5.64, \mathrm{P}=0.001)$. In the poor risk MSKCC cohort with SII index $>883$, the estimated mean PFS was $7.9 \pm 2.7$ months $(\mathrm{P}=0.001)$, while the estimated mean OS was $13.5 \pm 3.4$ months $(\mathrm{P}=0.012)$. Similarly, intermediate risk category with SII index $>883$ had an estimated mean PFS of $8.5 \pm 2.8$ months $(\mathrm{P}=0.014)$, while the estimated mean OS stood at $14.4 \pm 2.8$ months $(\mathrm{P}=0.036)$. Similarly, a high CAR was acted as a significant predictor of PFS (HR: 4.445, 95\% CI: $0.564-6.578, \mathrm{P}=0.002$ ) but not OS (HR: 1.002, 95\% CI: $0.076-1.763, \mathrm{P}=0.554$ ) (Table 2). The estimated mean OS for cases with $\mathrm{CAR} \leq 0.11$ was not significant at 16.9 months with SE being 3.74 and $95 \%$ CI of 15.1 - $18.8(\mathrm{P}=0.229)$ and the mean PFS for the same was significant at 12.9 months $(95 \% \mathrm{CI}: 10.8-15.1, \mathrm{SE}: 4.50, \mathrm{P}=$ $0.029)$. In the poor risk MSKCC cohort with CAR $>0.11$, the estimated mean PFS was $6.8 \pm 2.2$ months $(\mathrm{P}=0.031)$, while the estimated mean OS was $11.8 \pm 2.8$ months $(\mathrm{P}=0.046)$. Similarly, intermediate risk category with CAR $>0.11$ had an estimated mean PFS of $7.6 \pm 2.4$ months $(\mathrm{P}=0.004)$, while the estimated mean OS stood at $12.7 \pm 3.1$ months $(\mathrm{P}=0.026)$.

\section{Discussion}

Currently, the recognition of prognostic biomarkers focuses on a serum marker which represents the tumor's biological behav- ior but might not be a true indicator for the authentic burden of RCC. The linkage between inflammation and malignancy has been widely researched in the last two decades. Immune cells play a predominant role in the inflammatory process leading to the production of cytokines and chemokines that promote tumor growth, angiogenesis and metastasis [14]. Thereupon, the complex balance between inflammatory cells and substances produced by inflammation may influence the type of cells detectable in the peripheral circulation. The addition of the peripheral blood biomarkers as an essential array to existing prognosis model is helpful in channelizing clinical treatment strategy $[8,15,16]$. Since inflammation deeply affects cancer microenvironment that supports tumor progression, many studies evaluated the role of prognostic scores based on peripheral inflammation cells in several tumors, in particular RCC. Though the role of inflammatory blood parameters has found a way into the various cancer research studies off late, the coherence and importance of prognostic variables such as PLR, LMR, SII index and CAR still needs to be explored in the field of non-clear cell RCC which currently lacks any validated prognostic model. In the present analysis, we found that pretreatment PLR, LMR, SII index and CAR can be applied as determinants of PFS and OS for metastatic non-clear cell RCC. A high PLR, SII index along with CAR and a low LMR were correlated with poor PFS. To the contrary, a poor OS was correlated only with a high PLR and a low LMR but not with a high SII index and CAR. Moreover, a subgroup analysis by histology, T stage, FG, tumor size, TN, MI and (p) LN status was done to study the impact of PLR, LMR, SII index and $\mathrm{CAR}$ on the progression versus the non-progression groups. As testing for these cheap yet promising hematological biomarkers is easily accomplished in all clinical centers, these can act as a convenient yet game changing code for predicting the PFS and OS in cases of metastatic non-clear cell RCC.

The potential mechanisms of altered PLR and LMR affecting the prognostication of these uncommon metastatic RCC patients remain speculative at this juncture. Elevated platelet count is a common entity in patients with advanced malignancy, and is related to inferior oncologic upshot [17]. Platelets act as an important driver in tumor cell growth, angiogenesis and metastasis. Platelets usually protect the malignant cell from being detected by the autoimmune system by facilitating its adhesion to the vascular endothelium, or binding the malignant cells through its ligands [18]. A recent study [19] cultivated human platelets and ovarian tumor cells together, and proposed that synergy between platelet and malignant cells enhanced metastasis formation. They further proposed that a blockage of key platelet receptor sites crippled metastatic potential. Lymphocyte represents the cellular basis of immune surveillance and its penetration into the tumor microenvironment acts as a stealth mechanism for timely immune response against metastasis and tumor propagation [20, 21]. Lymphopenia acts as an intrinsic component of elevated PLR. A decreased lymphocyte count results in attenuation of immunologic antitumor response to a rampant tumor march. Nonetheless, monocytes bathing tumor clusters have a role in tumor development and progression [5]. Monocytes are closely associated with the creation of tumor-associated macrophages (TAMs). There is enough proof in literature to support the no- 

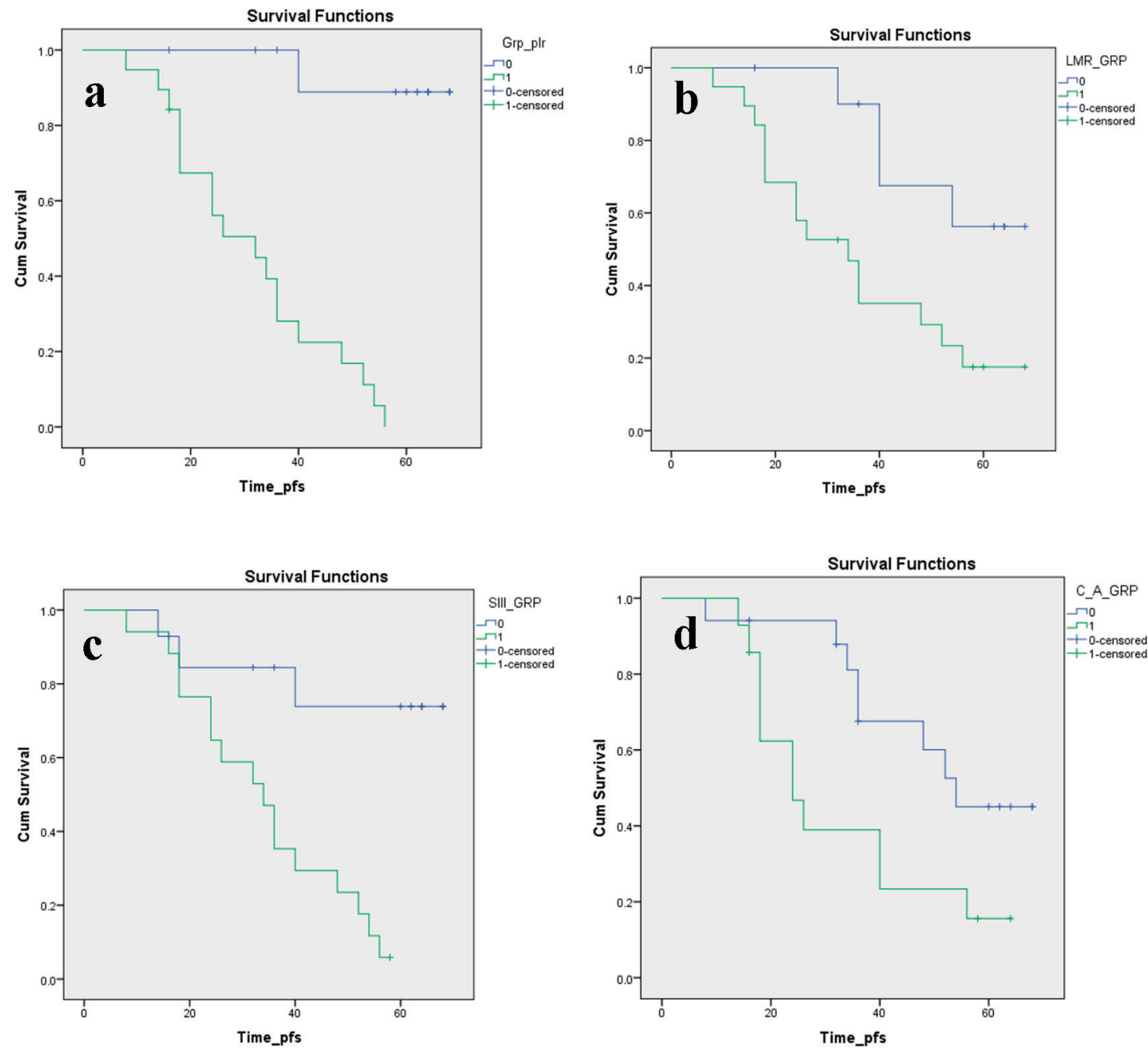

Figure 2. Kaplan-Meier plots illustrating PFS according to the (a) PLR, (b) LMR, (c) SII index and (d) CAR, respectively. PFS: progression-free survival; PLR: platelet to lymphocyte ratio; LMR: lymphocyte to monocyte ratio; SII: systemic immune inflammation; CAR: C-reactive protein/albumin ratio.

tion that the TAMs enhance tumor progression. Positioned on these facts, it is logical to believe that an elevated PLR and/or a decreased LMR lead to inferior survival.

The estimated PFS for the entire study cohort was 10.8 months $(\mathrm{P}<0.001)$ while the estimated OS for the same was 15.5 months $(\mathrm{P}=0.06)$. The actuarial PFS was only $5.6 \%$ at
14 months for patients with PLR above the cut-off mark of 171 with an estimated mean PFS of 7.7 months $(\mathrm{P}=0.0001)$ which amply showcased the utility of the PLR in prognostication. Only about one-third patients $(39.6 \%)$ survived without any progression at 9 months yet again confirming the severity of the PLR above the cut-off mark (Table 2, Fig. 2a). Simi-

Table 3. Estimated and Actuarial Survival Data for OS Based on Kaplan-Meier Survival Plots

\begin{tabular}{|c|c|c|c|c|c|c|c|}
\hline \multirow{2}{*}{ Variables } & \multicolumn{3}{|c|}{ Actuarial survival (\%) } & \multirow{2}{*}{$\begin{array}{l}\text { Estimated mean OS } \\
(95 \% \text { CI) (months) }\end{array}$} & \multirow{2}{*}{ SE } & \multirow{2}{*}{$\begin{array}{l}\text { Log rank (Mantel } \\
\text { Cox) coefficient }\end{array}$} & \multirow{2}{*}{ P value } \\
\hline & 9 months & 12 months & 14 months & & & & \\
\hline PLR & 66 & 49.5 & 39.6 & $12.6(10.2-14.9)$ & 2.47 & 10.83 & 0.001 \\
\hline SII index & 77.9 & 60.6 & 50.5 & $13.9(11.5-16.2)$ & 4.78 & 3.43 & 0.064 \\
\hline CAR & 57.1 & 52.3 & 47.6 & $13.5(10.3-16.6)$ & 6.51 & 1.45 & 0.229 \\
\hline
\end{tabular}

OS: overall survival; PLR: platelet to lymphocyte ratio; LMR: Iymphocyte to monocyte ratio; SII: systemic immune inflammation; CAR: C-reactive protein/albumin ratio; $\mathrm{Cl}$ : confidence interval; $\mathrm{SE}$ : standard error. 

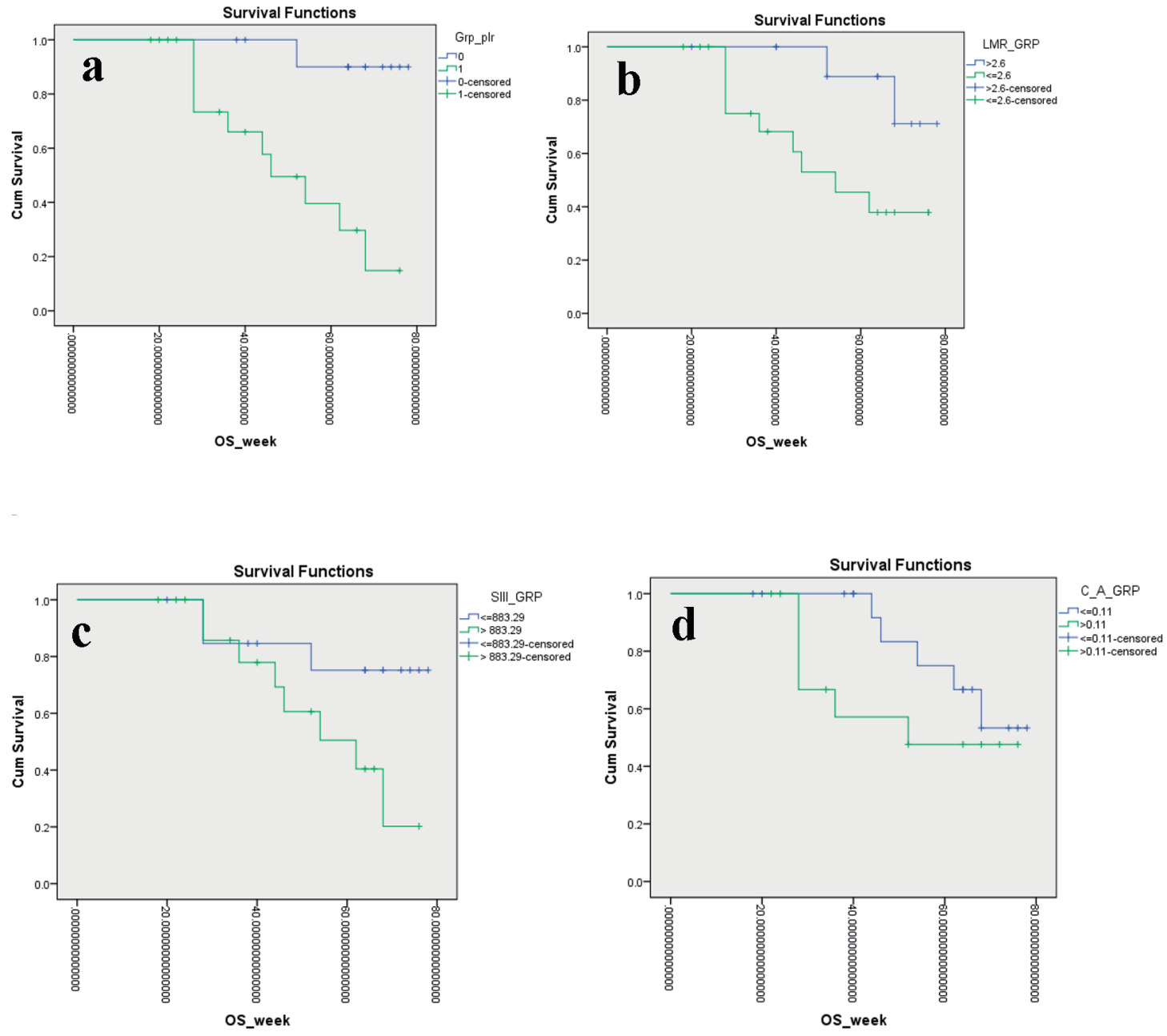

Figure 3. Kaplan-Meier plots illustrating OS according to the (a) PLR, (b) LMR, (c) SII index and (d) CAR, respectively. OS: overall survival; PLR: platelet to lymphocyte ratio; LMR: Iymphocyte to monocyte ratio; SII: systemic immune inflammation; CAR: C-reactive protein/albumin ratio.

larly, the actuarial OS stood at $39.6 \%$ at 14 months (for PLR $>$ 171) with a significant estimated mean OS of 12.6 months $(\mathrm{P}=$ $0.001)$ which further stressed on the importance of the PLR for the same (Table 3, Fig. 3a). Furthermore, the actuarial PFS was somewhat better $(17.5 \%$ at 14 months for patients with LMR $<2.61$ with an estimated mean PFS of 8.9 months $(\mathrm{P}=0.016))$ which aptly embodied the adequacy of the LMR in prognosti- cation. Only about one-third patients (35.1\%) survived without any progression at 9 months yet again confirming the severity of the LMR below the cut-off mark (Table 4, Fig. 2b). In addition, the actuarial OS stood at $45.5 \%$ at 14 months (for LMR $<2.61)$ with a significant estimated mean OS of 13.5 months $(\mathrm{P}=0.035)$ which further underlined on the importance of the LMR for the same (Table 3, Fig. 3b).

Table 4. Estimated and Actuarial Survival Data for PFS Based on Kaplan-Meier Survival Plots

\begin{tabular}{|c|c|c|c|c|c|c|c|}
\hline \multirow{2}{*}{ Variables } & \multicolumn{3}{|c|}{ Actuarial survival (\%) } & \multirow{2}{*}{$\begin{array}{l}\text { Estimated mean PFS } \\
\text { (95\% CI) (months) }\end{array}$} & \multirow{2}{*}{ SE } & \multirow{2}{*}{$\begin{array}{l}\text { Log rank (Mantel } \\
\text { Cox) coefficient }\end{array}$} & \multirow{2}{*}{ P value } \\
\hline & 9 months & 12 months & 14 months & & & & \\
\hline PLR & 39.3 & 16.8 & 5.6 & $7.8(6.1-9.4)$ & 2.93 & 22.10 & 0.0001 \\
\hline SII index & 35.3 & 23.5 & 5.9 & $8.5(6.8-10.3)$ & 3.63 & 10.73 & 0.001 \\
\hline CAR & 39.3 & 23.4 & 15.6 & $8.2(5.8-10.6)$ & 4.91 & 4.74 & 0.029 \\
\hline
\end{tabular}

PFS: progression-free survival; PLR: platelet to lymphocyte ratio; LMR: Iymphocyte to monocyte ratio; SII: systemic immune inflammation; CAR: Creactive protein/albumin ratio; Cl: confidence interval; SE: standard error. 
From previous umpteen numbers of studies, it has been validated that neutrophils can promote tumor seeding and adhesion through the secretion of circulating growth factors [22]. Neutrophil to lymphocyte ratio has stood the test of time and is probably the most prolific prognostic index associated with prognosis in malignancy of lung, pancreatic, breast, colorectal, gastric and kidney $[16,23]$. Decreased lymphocyte count in preoperative blood investigation was associated with poor prognosis in patients with RCC [24]. As discussed before, a high platelet score was also associated with poor prognosis in RCC. SII index incorporated these three parameters and has been already significantly associated with prognosis in hepatocellular and colorectal malignancy. The actuarial PFS was again a dismal $5.9 \%$ at 14 months for patients with SII index $>883$ with an estimated mean PFS of 8.5 months $(\mathrm{P}=0.001)$ which again abundantly signified the utility of the SII index in prognostication. Only about one-third patients $(35.3 \%)$ survived without any progression at 9 months yet again confirming the severity of the SII index above the cut-off mark (Table 4, Fig. 2c). Similarly, the actuarial OS stood at $50.5 \%$ at 14 months (for SII index > 883) with a non-significant estimated mean OS of 13.9 months $(\mathrm{P}=0.064)$ for the same (Table 3 , Fig. 3c). Therefore, the integrated use of SII index and related parameters might lead to a significant advancement in therapeutic monitoring of patients with metastatic non-clear cell RCC, even if prospective studies are needed to further validate this observation.

Although the baseline CRP level is affected by environmental and genetic factors, it is produced mainly by hepatocytes and is regulated by proinflammatory cytokines (IL-6) [25]. The potential mechanisms for the association of CRP with malignancy have been proposed as tissue inflammation caused by the tumor growth or as an immune response to tumor antigens. A recent study showed that activity of the IL-6/ CRP network in RCC patients endows to the acute-phase reaction in local inflammatory response [26]. Other clinical analysis also showed that increased CRP level stands glove in hand with poorer OS of RCC patients and CRP has a significant impact on OS of metastatic RCC patients [27].

Hypoalbuminemia is an easy, reproducible biomarker which is closely correlated with other parameters of nutritional status. Furthermore, serum albumin as a biomarker of protein-energy malnutrition can provide vital information that supplements BMI and changes in body weight, which may at times not accurately reflect the nutritional status due to normal limits [28]. Protein deficiency can lead to significant impairment in organ function and immunosuppression. In addition, hypoalbuminemia before surgery was associated with higher mortality in patients with underwent surgery for RCC [29]. In recent times, CAR has been used to predict the prognosis of several cancers and this is the first report in this part of the world showing that CAR can predict the PFS of metastatic non-clear cell RCC undergoing CNT.

The actuarial PFS was $15.6 \%$ at 14 months for patients with CAR $>0.11$ with an estimated mean PFS of 8.2 months $(P=0.029)$ which again abundantly verified the utility of the CAR in prognostication. A slightly more number of patients than previous one-third cases in aforementioned variables $(39.3 \%)$ survived without any progression at 9 months yet again confirming the vitality of the CAR above the cut-off mark (Table 4, Fig. 2d). Similarly, the actuarial OS stood at $47.6 \%$ at 14 months (for CAR $>0.11$ ) with a non-significant estimated mean OS of 13.5 months $(P=0.229)$ for the same (Table 3, Fig. 3d).

The entire cohort PFS and OS (at 14 months) for the MSK$\mathrm{CC}$ intermediate risk group were 8.2 months $(\mathrm{P}=0.07)$ and 12.5 months $(P=0.12)$, respectively. Similarly, the PFS and OS (at 14 months) for the poor risk group were 7.7 months ( $P$ $=0.04)$ and 13.8 months $(\mathrm{P}=0.03)$, respectively. Our findings have some clinical implications. Firstly, compared with the postoperative HPE report, preoperative circulating biomarkers are more effective and suitable prognostic indicators in patients with metastatic non-clear cell RCC. Secondly, according to the preoperative biomarker level, patients with high risk can be selected for extensive LN dissection and further adjuvant treatment in form of some clinical trial. Thirdly, our result can be used to stratify patients who are more likely to respond to biomarker-based enrichment strategy in future clinical trials.

Our study also has several limitations. Firstly, it is a retrospective and single-center study, which may limit the prognostic value for the biomarkers in question. Therefore, a largescale prospective validation study is needed. Secondly, several factors which could affect inflammation such as life style and smoking status were not assessed in the study. Last but most important, PFS was recorded based on radio-examination which may be longer than the actual PFS as some patients admitted to hospital only when they had obvious symptoms. In summary, this study demonstrated that preoperative PLR, LMR, SII index and CAR are an independent predictor of PFS and OS (PLR and LMR only) for patients with metastatic nonclear cell RCC and can be used to predict the outcome patients undergoing CNT.

\section{Conclusions}

The use of validated prognostic indices is essential in clinical practice to better make correct decisions on the use of high end and extensive surgeries (CNT) and to potentially reduce the impact of comorbidities especially in more frail patients. In metastatic non-clear cell RCC, there is currently no acceptable protocol for guiding decision for CNT and adjuvant treatment. In this analysis, we want to purpose preoperative biomarkers such as PLR, LMR, SII index, CAR and SST as a new tool to define PFS and OS stratification in metastatic non-clear cell RCC patients. These innovative yet cheap biomarkers can predict response to treatment and clinical outcome of these patients, giving a potential simple tool to monitor the effect of treatment on the clinical outcome of these patients. In addition, this inflammatory prognostic model is of special interest in metastatic non-clear cell RCC, which is a work in progress and new immune-oncologic agents, like checkpoint inhibitors and some tyrosine kinase inhibitors (TKIs) are in active development as agents for these cases. In conclusion, to our limited knowledge, this is the first study of its magnitude and kind in this part of the world to demonstrate that these biomarkers could represent an independent prognostic factor for patients with metastatic non-clear cell RCC undergoing CNT. Valida- 
tion in a larger prospective dataset is desirable.

\section{Acknowledgments}

None.

\section{Financial Support}

None.

\section{Conflict of Interest}

None.

\section{Informed Consent}

Not applicable.

\section{Author Contributions}

Dr Sasanka Kumar Barua conceptualized the research work and method framework with special emphasis on metastatic non-clear cell RCC and wrote the paper. Dr Yashasvi Singh did the data entry, statistical analysis and served as the corresponding author. Dr SaumarJyoti Baruah and Dr Rajeev T. Puro helped in the comparison of the research analysis with MSKCC data. Dr Puskal Kumar Bagchi helped in writing the paper and statistical analysis. Dr Debanga Sarma and Dr Mandeep Phukan helped in writing the paper and formulate data entry.

\section{References}

1. Rini BI, Campbell SC, Escudier B. Renal cell carcinoma. Lancet. 2009;373(9669):1119-1132.

2. Pal SK, Choueiri TK. Systemic therapies for metastatic renal cell carcinoma of variant histology. In: Renal Cell Carcinoma, Tannir NM (Ed), Oxford American Oncology Library, New York; 2014. 2. .

3. Ljungberg B, Bensalah K, Canfield S, Dabestani S, Hofmann F, Hora M, Kuczyk MA, et al. EAU guidelines on renal cell carcinoma: 2014 update. Eur Urol. 2015;67(5):913-924.

4. Albiges L, Choueiri T, Escudier B, Galsky M, George D, Hofmann F, Lam T, et al. A systematic review of sequencing and combinations of systemic therapy in metastatic renal cancer. Eur Urol. 2015; 67(1):100-110.

5. Mantovani A, Allavena P, Sica A, Balkwill F. Cancerrelated inflammation. Nature. 2008;454(7203):436-444.

6. Aggarwal BB, Vijayalekshmi RV, Sung B. Targeting inflammatory pathways for prevention and therapy of cancer: short-term friend, long-term foe. Clin Cancer Res.
2009;15(2):425-430.

7. Grivennikov SI, Greten FR, Karin M. Immunity, inflammation, and cancer. Cell. 2010;140(6):883-899.

8. Templeton AJ, Ace O, McNamara MG, Al-Mubarak M, Vera-Badillo FE, Hermanns T, Seruga B, et al. Prognostic role of platelet to lymphocyte ratio in solid tumors: a systematic review and meta-analysis. Cancer Epidemiol Biomarkers Prev. 2014;23(7):1204-1212.

9. Gu L, Li H, Chen L, Ma X, Li X, Gao Y, Zhang Y, et al. Prognostic role of lymphocyte to monocyte ratio for patients with cancer: evidence from a systematic review and meta-analysis. Oncotarget. 2016;7(22):31926-31942.

10. Hu B, Yang XR, Xu Y, Sun YF, Sun C, Guo W, Zhang $\mathrm{X}$, et al. Systemic immune-inflammation index predicts prognosis of patients after curative resection for hepatocellular carcinoma. Clin Cancer Res. 2014;20(23):62126222.

11. Steffens S, Kohler A, Rudolph R, Eggers H, Seidel C, Janssen M, Wegener G, et al. Validation of CRP as prognostic marker for renal cell carcinoma in a large series of patients. BMC Cancer. 2012;12:399.

12. Yasuda Y, Saito K, Yuasa T, Kitsukawa S, Urakami S, Yamamoto S, Yonese J, et al. Prognostic impact of pretreatment C-reactive protein for patients with metastatic renal cell carcinoma treated with tyrosine kinase inhibitors. Int J Clin Oncol. 2013;18(5):884-889.

13. Lopez-Lago MA, Posner S, Thodima VJ, Molina AM, Motzer RJ, Chaganti RS. Neutrophil chemokines secreted by tumor cells mount a lung antimetastatic response during renal cell carcinoma progression. Oncogene. 2013;32(14):1752-1760.

14. Tsao CK, Small AC, Kates M, Moshier EL, Wisnivesky JP, Gartrell BA, Sonpavde G, et al. Cytoreductive nephrectomy for metastatic renal cell carcinoma in the era of targeted therapy in the United States: a SEER analysis. World J Urol. 2013;31(6):1535-1539.

15. Wang Z, Peng S, Wang A, Xie H, Guo L, Jiang N, Niu Y. Platelet-lymphocyte ratio acts as an independent predictor of prognosis in patients with renal cell carcinoma. Clin Chim Acta. 2018;480:166-172.

16. Templeton AJ, McNamara MG, Seruga B, Vera-Badillo FE, Aneja P, Ocana A, Leibowitz-Amit R, et al. Prognostic role of neutrophil-to-lymphocyte ratio in solid tumors: a systematic review and meta-analysis. J Natl Cancer Inst. 2014;106(6):dju124.

17. Gu L, Li H, Gao Y, Ma X, Chen L, Li X, Zhang Y, et al. The association of platelet count with clinicopathological significance and prognosis in renal cell carcinoma: a systematic review and meta-analysis. PLoS One. 2015;10(5):e0125538.

18. Heng DY, Xie W, Regan MM, Warren MA, Golshayan AR, Sahi C, Eigl BJ, et al. Prognostic factors for overall survival in patients with metastatic renal cell carcinoma treated with vascular endothelial growth factor-targeted agents: results from a large, multicenter study. J Clin Oncol. 2009;27(34):5794-5799.

19. Orellana R, Kato S, Erices R, Bravo ML, Gonzalez P, Oliva B, Cubillos S, et al. Platelets enhance tissue factor protein and metastasis initiating cell markers, and act as 
chemoattractants increasing the migration of ovarian cancer cells. BMC Cancer. 2015;15:290.

20. Hoffmann TK, Dworacki G, Tsukihiro T, Meidenbauer N, Gooding W, Johnson JT, Whiteside TL. Spontaneous apoptosis of circulating $\mathrm{T}$ lymphocytes in patients with head and neck cancer and its clinical importance. Clin Cancer Res. 2002;8(8):2553-2562.

21. Dunn GP, Old LJ, Schreiber RD. The immunobiology of cancer immunosurveillance and immunoediting. Immunity. 2004;21(2):137-148.

22. Sun L, Li D, Hemraz UD, Fenniri H, Webster TJ. Selfassembled rosette nanotubes and poly(2-hydroxyethyl methacrylate) hydrogels promote skin cell functions. J Biomed Mater Res A. 2014;102(10):3446-3451.

23. Templeton AJ, Knox JJ, Lin X, Simantov R, Xie W, Lawrence $\mathrm{N}$, Broom $\mathrm{R}$, et al. Change in neutrophil-to-lymphocyte ratio in response to targeted therapy for metastatic renal cell carcinoma as a prognosticator and biomarker of efficacy. Eur Urol. 2016;70(2):358-364.

24. Saroha S, Uzzo RG, Plimack ER, Ruth K, Al-Saleem T. Lymphopenia is an independent predictor of inferior outcome in clear cell renal carcinoma. J Urol. 2013;189(2):454-461.
25. Greenfield JR, Samaras K, Jenkins AB, Kelly PJ, Spector TD, Gallimore JR, Pepys MB, et al. Obesity is an important determinant of baseline serum $\mathrm{C}$-reactive protein concentration in monozygotic twins, independent of genetic influences. Circulation. 2004;109(24):3022-3028.

26. Deichmann M, Benner A, Waldmann V, Bock M, Jackel A, Naher H. Interleukin-6 and its surrogate C-reactive protein are useful serum markers for monitoring metastasized malignant melanoma. J Exp Clin Cancer Res. 2000;19(3):301-307.

27. Fujita T, Nishi M, Tabata K, Matsumoto K, Yoshida $\mathrm{K}$, Iwamura M. Overall prognostic impact of C-reactive protein level in patients with metastatic renal cell carcinoma treated with sorafenib. Anticancer Drugs. 2016;27(10):1028-1032.

28. Baker JP, Detsky AS, Wesson DE, Wolman SL, Stewart S, Whitewell J, Langer B, et al. Nutritional assessment: a comparison of clinical judgement and objective measurements. N Engl J Med. 1982;306(16):969-972.

29. Kim HL, Belldegrun AS, Freitas DG, Bui MH, Han KR, Dorey FJ, Figlin RA. Paraneoplastic signs and symptoms of renal cell carcinoma: implications for prognosis. J Urol. 2003;170(5):1742-1746. 